\title{
Optimal Green Energy Utilization in MIMO Systems with Hybrid Energy Supplies
}

\author{
Congshi Hu, Jie Gong, Member, IEEE, Xiaolei Wang, Student Member, IEEE, Sheng Zhou, Member, IEEE \\ and Zhisheng Niu, Fellow, IEEE
}

\begin{abstract}
This paper considers the power allocation of a single Multi-Input Multi-Output (MIMO) wireless link with hybrid energy harvesting and grid power supply. We optimize the power allocation to minimize the power grid energy consumption while guaranteeing users' QoS, which is defined as transmitting a certain number of bits in finite time horizon. First we solve the offline throughput maximization problem and propose the optimal solution called two-stage spatial-temporal water-filling, which allocates harvested energy in the first stage and then power grid energy in the second stage. Based on this, we solve the offline power grid energy minimization problem by utilizing its equivalence to the throughput maximization problem. Then, we use the Markov Decision Process (MDP) to get the optimal online power grid energy minimization policy. Furthermore, we propose two near-optimal algorithms with reduced complexity, namely the constant water level algorithm and the bit-aware water level algorithm. We evaluate the performance of the proposed offline and online algorithms by numerical simulations, showing that the bit-aware water level algorithm performs closely to the optimal online solution.
\end{abstract}

Index Terms-Green communications, energy harvesting, MIMO systems, two-stage spatial-temporal water-filling, MDP.

\section{INTRODUCTION}

$\mathbf{I}$ $\mathrm{N}$ wireless cellular networks, base stations (BSs) consume more than 50 percent of the power [1]. With increasing number of BSs, how to reduce the power consumption in BSs plays an important role for realizing green communications. A traditional way to reduce power consumption is to design energy-efficient communication protocols using power grid energy only, such as BSs sleeping [2]. These methods are effective when there is low traffic in the network because the most intuitive idea is to switch off some BSs during the offpeak hours. However, if the traffic is intense, few BSs can be switched off and these sleeping based algorithms may not be effective. Recently, with the development of energy harvesting technologies, it is feasible for BSs to be powered by green energy, such as solar energy and wind energy. For instance, Ericsson has developed a wind-powered tower for wireless BSs [3]. The renewable energy provides a promising option to

Copyright (c) 2014 IEEE. Personal use of this material is permitted. However, permission to use this material for any other purposes must be obtained from the IEEE by sending a request to pubs-permissions@ieee.org.

The authors are with Tsinghua National Laboratory for Information Science and Technology, Department of Electronic Engineering, Tsinghua University, Beijing 100084, P. R. China. Correspondence should be made to Sheng Zhou at: sheng.zhou@tsinghua.edu.cn

Part of this paper has been presented at IEEE ICCC'13 [24]. The research work is partially sponsored by the National Basic Research Program of China (973Green: No. 2012CB316001); by the Nature Science Foundation of China (No. 61201191, No. 61021001); and by Hitachi R\&D Headquarter. save the power grid energy and reduce $\mathrm{CO}_{2}$ emissions. In the energy harvesting systems, BSs can harvest energy freely from random energy sources. However, because of the dynamic energy arrival process, it is often very difficult to guarantee sufficient power supplies for BSs by only using harvested energy. Hence, we envision that future BSs are co-powered by both the power grid energy and the harvested energy. The BSs can use harvested energy stored in the battery or the power grid energy or both of them. In addition, the variation of the harvested energy both in the time domain and the space domain leads to new challenges in such co-powered BSs. Specifically, the Multi-Input Multi-Output (MIMO) antenna system has been widely used in modern BSs, but how to utilize the harvested energy efficiently in it is still an open issue. A desirable algorithm is to minimize the power grid energy while guaranteeing users' quality of service (QoS), which is defined as transmitting a certain number of bits before a given deadline.

As mentioned above, the variation of harvested energy both in the time domain and the space domain leads to challenging issues. In addition, the MIMO technology has drawn much academic attention and become an emerging technology. It not only brings capacity gain but also reduces the power consumption dramatically [21], thus is a key technology in modern BSs. As a result, it is desirable to consider how to allocate power with MIMO systems in BSs powered by power grid and harvested energy. And in this paper, we optimize the energy utilization in such systems by minimizing the power grid energy consumption while guaranteeing that all the bits can be transmitted before a given deadline. We begin with discussing the throughput maximization problem in the energy harvesting and power grid coexisting MIMO system under the offline condition with non-causal full information. Based on this, we propose the optimal offline grid power minimization policy by utilizing its equivalence to the throughput maximization problem. And for the online condition with causal information, the optimal policy can be achieved by MDP. For the purpose of practical application with reduced complexity, we also propose some near-optimal online solutions based on the insights from the offline solution. The main contributions are presented as follows.

- For the offline throughput maximization problem, we show that it is a convex optimization problem and the global optimal solution can be achieved. Considering the energy causality and the limited battery capacity, we propose the two-stage spatial-temporal water-filling 
solution to find the optimal power allocation. Specifically, we allocate the harvested energy in the first stage and then the power grid energy in the second stage. As for the harvested energy allocation in the first stage, it is allocated first in the spatial domain by the traditional water-filling and then adjusted in the temporal domain by the directional water-filling. Note that these two domains are not completely separated because when we adjust the temporal water level, we must re-consider the energy and channel condition for each antenna to keep different antennas in the same slot maintain the same water level.

- For the power grid energy minimization problem under the non-causal full information condition, it can also be solved by convex optimization. However in this paper, we solve this minimization problem by showing it to be a dual problem of the throughput maximization problem. This comes from the reason that the throughput maximization problem has a clearer architecture and is easier to be implemented. In addition, for the power grid energy minimization problem under the online condition with causal information, we give the optimal solution by using MDP [22].

- We propose two near-optimal online solutions with reduced complexity based on the insights from the offline solution for practical applications. The optimal offline solution structure tells us that the energy level tries to be constant but needs some adjustment due to the energy causality. Hence, the constant water level algorithm and the bit-aware water level algorithm are proposed. We evaluate them by numerical results, showing the bit-aware water level performs better and is close to the optimal MDP solution.

The rest of the paper is organized as follows. Related work on the energy harvesting issues is discussed in Section II. Section III presents the model of the energy harvesting and power grid coexisting MIMO system. The offline throughput maximization problem is studied in Section IV, where the optimal two-stage spatial temporal water filling algorithm is proposed and illustrated by some examples. Then the power grid energy minimization problem is analyzed in Section $\mathrm{V}$ and we present how to solve it by using its dual property of the throughput maximization. In Section VI, we consider the online policies. The optimal online solution can be achieved by MDP and two near-optimal polices with reduced complexity are given based on the optimal offline structure. Numerical results are presented in Section VII to verify the performance of the proposed algorithms. And finally, Section VIII concludes all this work. For the ease of presence, we give the key notations in Table I.

\section{RELATED WORK}

In the literature, there have been a lot of researches on energy harvesting issues and the power allocation is one of the hottest topics. For the online case with causal information where only the current and past energy and channel conditions can be known, power allocation policies with energy harvesting transmitter are studied in [7] - [9]. The crosslayer resource management for wireless networks operating with rechargeable batteries under general arrival was studied in [7] and the authors designed decoupled admission control and power allocation decisions using Lyapunov optimization to achieve asymptotic optimality. Ref. [8] identified throughput optimal and mean delay optimal energy management policies and showed a greedy policy to be optimal in low SNR regime. And a throughput maximization algorithm in point-to-point communications with causal information was proposed in [9] using Markov Decision Process (MDP). Although these causal information based policies are proposed, the properties of the optimal solution can not be directly obtained. Recently, some research efforts have been put on studying the structure of the optimal power allocation under the offline condition with noncausal full information where all the future energy conditions can be known before transmission [10] - [13]. In [10], the authors presented the optimal transmission policy to maximize the short-term throughput with finite battery capacity and found the relation between the throughput maximization problem and the completion time minimization problem. Refs. [11] - [13] considered using directional water-filling power allocation algorithm in different channel models including the Gaussian fading channel [11], the broadcast channel [12] and the multiple access channel [13] to maximize the throughput in finite time horizon. Besides, there are some other papers on the offline power allocation to optimize the energy efficiency. In [14], the authors devised a lazy algorithm that varied transmission times according to backlog and showed it to be more energy efficient than a deterministic schedule. Ref. [15] investigated the properties of the optimal offline scheduling for packets and showed that when packet interarrival times were identically and independently distributed (i.i.d.), the resulting optimal transmission durations of packets exhibited a symmetry property. Ref. [16] studied the strict QoS constraints, such as individual packet deadlines, finite buffer and so on, and proposed a general calculus approach. But still, these offline solutions mainly focus on the single antenna scenario.

There are also some papers considering the hybrid energy supplies scenario. In [17], the authors proposed a twotimescale delay-optimal base station discontinuous transmission control and user scheduling for energy harvesting downlink coordinated MIMO networks. But different from what we will talk in this paper, the authors in this paper focused on the delay-aware problem in the energy harvesting MIMO system and characterized the sufficient conditions for stable data queues. In [18] of the green energy utility optimization problem, the authors decomposed it into the multi-stage energy allocation problem and the multi-BSs energy balancing problem to get the optimal policy. Furthermore, recently we have also analyzed the optimal power allocation for energy harvesting and power grid coexisting wireless communication systems and proposed the reverse multi-stage water filling (WF) algorithm for random data arrivals [19].

\section{SYSTEM MODEL}

We consider a single wireless MIMO link as shown in Fig. 1 , where the transmitter and the receiver are equipped with $n_{t}$ 
TABLE I

NOTATIONS IN THE PAPER

\begin{tabular}{|l|l|}
\hline Notations & \\
\hline $\mathbb{C}$ & Set of complex numbers \\
$\operatorname{tr}(\mathbf{A})$ & Trace of matrix $\mathbf{A}$ \\
$\mathbf{A}^{\mathrm{H}}$ & Conjugate transpose matrix of $\mathbf{A}$ \\
$n_{t}$ & Number of transmit antennas \\
$n_{r}$ & Number of receive antennas \\
$\mathbf{X}_{i}$ & Transmitted signal vector in slot $i$ \\
$\mathbf{Y}_{i}$ & Received signal vector in slot $i$ \\
$\mathbf{H}_{i}$ & Channel fading matrix in slot $i$ \\
$\mathbf{Q}_{i}$ & The auto-covariance of $\mathbf{X}(i)$ \\
$p_{G, i}, p_{H, i}$ & Power grid energy, harvested energy used in slot $i$ \\
$p_{i}(j)$ & Power of antenna $m$ in slot $i$ \\
$E_{i}$ & Harvested energy in time slot $i$ \\
$e_{i}$ & remaining battery energy before slot $i$ \\
$\lambda_{i}^{(j)}$ & The $m$ th singular value of $\mathbf{H}_{i}$ \\
$\mathbf{U}$ & Eigenmatrix of $\mathbf{H}^{\mathrm{H}} \mathbf{H}$ \\
\hline
\end{tabular}

and $n_{r}$ antennas respectively. The transmitter is powered by both harvested energy and power grid energy. The harvested energy is stored in a battery whose capacity is $E_{\max }$ and power grid is used if the harvested energy is insufficient. We assume that both the harvested energy and power grid energy are used only for transmission, which means that the processing energy is ignored. Note that there is only one battery in our system, which is used to store the harvested energy only. While for the grid power, it can be used directly from the grid power. And in fact, the real energy harvesting base stations have traditional power grid interface [20]. We assume that the battery is used to store the harvested energy only, while the grid power is directly drawn from the grid when necessary.

In other words, we consider the hardware limitation from two parts. For the power grid energy, the limitation is the average energy consumption. While for the harvested energy, the limitation lies on the finite battery capacity, which limits the the maximum available harvested energy.

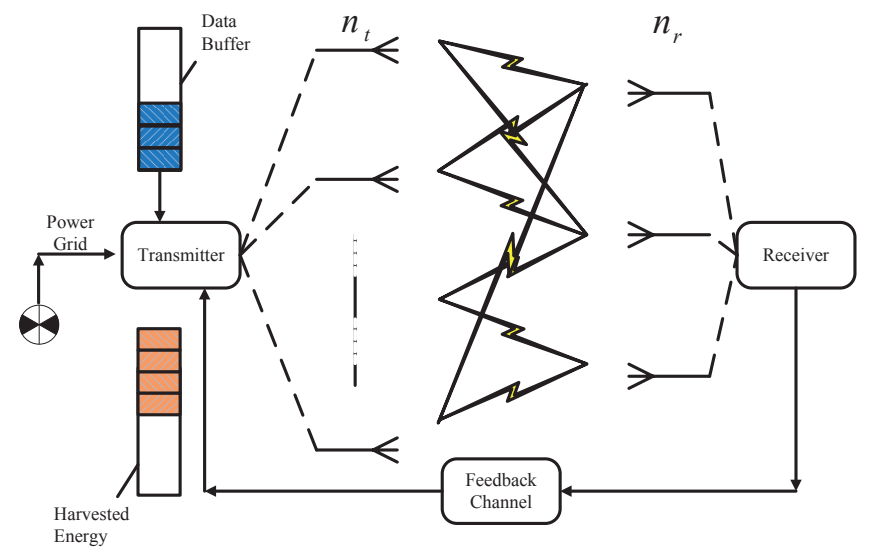

Fig. 1. The hybrid energy harvesting and power grid MIMO system model

We consider a time-slotted system with slot length $T_{f}$ and study the power allocation problem in finite $N$ slots. In each slot, the channel keeps constant, which means the block fading model is used. The received signal $\mathbf{Y}_{i} \in \mathbb{C}^{n_{r} \times 1}$ in slot $i \in$
$\{1, \ldots, N\}$ is given by

$$
\mathbf{Y}_{i}=\mathbf{H}_{i} \mathbf{X}_{i}+\mathbf{n}_{i},
$$

where $\mathbf{H}_{i} \in \mathbb{C}^{n_{r} \times n_{t}}$ is the channel state matrix, $\mathbf{X}_{i} \in \mathbb{C}^{n_{t} \times 1}$ is the transmitted signal vector and $\mathbf{n}_{i}$ is additive white Gaussian noise with zero mean and unit variance. Then the instantaneous rate $r_{i}$ in bits per channel use is [23]

$$
r_{i}=\frac{1}{2} \log \operatorname{det}\left[\mathbf{I}_{n_{r}}+\mathbf{H}_{i} \mathbf{Q}_{i} \mathbf{H}_{i}^{\mathrm{H}}\right],
$$

where $\mathbf{Q}_{i}$ is the covariance of transmitted signal vector, i.e.,

$$
\mathbf{Q}_{i}=\mathbf{X}_{i} \mathbf{X}_{i}^{\mathrm{H}} .
$$

The power allocated to antenna $j \in 1, \ldots, n_{t}$ is denoted as $p_{i}(j)$, which is the $j$ th diagonal element of $\mathbf{Q}_{i}$. Then, we know that the total transmission power denoted as $p_{i}$ is the trace of matrix $\mathbf{Q}_{i}$, i.e.,

$$
p_{i}=\operatorname{tr}\left[\mathbf{X}_{i} \mathbf{X}_{i}^{\mathrm{H}}\right] .
$$

In each slot, the total power $p_{i}$ consists the harvested power part, denoted as $p_{H, i}$, and the grid power part, denoted as $p_{G, i}=p_{i}-p_{H, i}$. And the harvested energy $\left\{E_{0}, E_{1}, \ldots, E_{N-1}\right\}$ arrives at the beginning of each slot.

There are two constraints on the harvested energy $p_{H, i}$ because of the energy causality and the finite battery capacity.

For the energy causality, the harvested energy can not be consumed before its arrival, which can be expressed as

$$
\sum_{i=1}^{k} T_{f} p_{H, i} \leq \sum_{i=0}^{k-1} E_{i}, k=1,2, \ldots, N .
$$

The second constraint is the finite battery capacity. We want to make sure that the reserved energy plus the harvested energy can not exceed the battery capacity. Because the data rate can be increased if the energy is used in advance instead of overflowed. So,

$$
\sum_{i=0}^{k} E_{i}-\sum_{i=1}^{k} T_{f} p_{H, i} \leq E_{\max }, k=1,2, \ldots, N-1 .
$$

On the other hand, the power grid energy can be used freely on the condition that the average power over all the $N$ slots is no larger than the threshold, denoted as $P_{G \text {,ave }}$. Our objective is to minimize the energy consumed by the power grid, which can be expressed as,

$$
\min \sum_{i=1}^{N} T_{f}\left(p_{i}-p_{H, i}\right) .
$$

Here we give a clear concept about the offline condition with non-causal full information and the online condition with causal full information using the mathematic notations introduced above. We call it the offline condition where all the channel matrices $\mathbf{H}_{i}(i=1, \ldots, N)$ and all the harvested energy arrivals $E_{i}(i=1, \ldots, N)$ can be known before the first slot. And if in slot $j$, only the current and past channel matrices $\mathbf{H}_{i}(i=1, \ldots, j)$ and harvested arrivals $E_{i}$ $(i=1, \ldots, j)$ can be known, we call it the online condition. First we analyze the offline problem because it is easy to see its optimal solution structure and can help to give some 
interesting insights. As mentioned before, we first solve the throughput maximization problem instead of solving the power grid energy minimization problem directly.

\section{OfFline Throughput Maximization}

In this section, we consider the greedy data source, which means that the data is always available at the transmitter. The objective is to maximize the throughput in the fading channel, which means that given the energy harvesting process and the average power grid energy constraint, we want to transmit as many bits as possible in the finite $N$ slots. We study the offline problem to find out the structure of the optimal policy.

Technically speaking, for the throughput maximization problem, the power grid and energy harvesting coexisting MIMO system is a special case of the MIMO system with energy harvesting powered only. Because the total power grid energy can be viewed as the initial battery energy, i.e., $E_{0}=N T_{f} P_{G \text {,ave }}$. But as mentioned before, our ultimate goal is to design the optimal scheduling to minimize the power grid energy consumption. So we still need to consider this coexisting scenario to know the required average power grid energy in future analysis.

\section{A. Problem Formulation}

Under the offline condition, the transmitter knows all the future system states, i.e., the energy arrivals and the channel states. Our goal is to maximize the number of bits transmitted in the $N$ time slots subject to the energy causality, the battery capacity and the grid power constraints. The problem is written as

Problem A:

$$
\begin{array}{cl}
\max & \sum_{i=1}^{N} \frac{T_{f}}{2} \log \left[\operatorname{det}\left(\mathbf{I}_{n_{r}}+\mathbf{H}_{i} \mathbf{Q}_{i} \mathbf{H}_{i}^{\mathrm{H}}\right)\right] \\
\text { s.t. } & (5) \text { and }(6), \\
& p_{H, k} \leq p_{k}, \forall k, \\
& p_{H, k} \geq 0, \forall k, \\
& \frac{1}{N} \sum_{i=1}^{N} p_{G, i}=\frac{1}{N} \sum_{i=1}^{N}\left(p_{i}-p_{H, i}\right) \leq P_{G, \text { ave }},
\end{array}
$$

where the constraints (9) and (10) guarantee that the harvested energy and grid power are both non-negative and constraint (11) implies the limited average grid power. Note that the constraint (5) with $K=N$ and (11) must be satisfied with equality for optimization. Otherwise, we can always get higher throughput by increasing the power $P_{H, N}$ without conflicting any constraint. For the offline condition, note that the unknown parameters in the Problem A are only $p_{i}, p_{H, i}(i=1, \ldots, N)$. Thus this problem is a deterministic optimization problem.

Using the determinant identity that $\operatorname{det}(\mathbf{I}+\mathbf{A B})=$ $\operatorname{det}(\mathbf{I}+\mathbf{B A})$, the objective function can be rewritten as,

$$
\log \operatorname{det}\left(\mathbf{I}+\mathbf{H} \mathbf{Q} \mathbf{H}^{\mathrm{H}}\right)=\log \operatorname{det}\left(\mathbf{I}+\mathbf{Q} \mathbf{H}^{\mathrm{H}} \mathbf{H}\right) .
$$

Since $\mathbf{H}^{\mathrm{H}} \mathbf{H}$ is Hermitian, it can be diagonalized as $\mathbf{H}^{\mathrm{H}} \mathbf{H}=$ $\mathbf{U}^{\mathrm{H}} \boldsymbol{\Lambda} \mathbf{U}$ with unitary $\mathbf{U}$ and non-negative diagonal matrix $\boldsymbol{\Lambda}=$ $\operatorname{diag}\left(\lambda_{1}, \ldots, \lambda_{M}, 0, \ldots, 0\right)$, where $M=\min \left(n_{t}, n_{r}\right)$.
As a result, the problem A can be reformulated as

Problem B:

$$
\begin{aligned}
\max _{\forall \widetilde{\mathbf{Q}}_{i} \in \Omega} \quad & \sum_{i=1}^{N} \frac{T_{f}}{2} \log \left\{\operatorname{det}\left[\mathbf{I}+\boldsymbol{\Lambda}_{i}^{\frac{1}{2}} \widetilde{\mathbf{Q}}_{i} \boldsymbol{\Lambda}_{i}^{\frac{1}{2}}\right]\right\}, \\
\text { s.t. } \quad & (5) \text { and }(6), \\
& p_{H, k} \leq p_{k}, \forall k, \\
& p_{H, k} \geq 0, \forall k, \\
& \frac{1}{N} \sum_{i=1}^{N}\left[\operatorname{tr}\left(\widetilde{\mathbf{Q}}_{i}\right)-p_{H, i}\right] \leq P_{G, \text { ave }}, \\
& \tilde{p}_{i}(j) \geq 0, \forall i \leq N, \forall j \leq n_{t},
\end{aligned}
$$

where $\mathbf{U}_{i}$ is the eigenmatrix of $\mathbf{H}_{i}^{\mathrm{H}} \mathbf{H}_{i}$, i.e., $\mathbf{H}_{i}^{\mathrm{H}} \mathbf{H}_{i}=$ $\mathbf{U}_{i}^{\mathrm{H}} \boldsymbol{\Lambda}_{i} \mathbf{U}_{i}$, and $\widetilde{\mathbf{Q}}_{i}=\mathbf{U}_{i} \mathbf{Q}_{i} \mathbf{U}_{i}^{\mathrm{H}}$ whose $j$ th diagonal element is denoted as $\tilde{p}_{i}(j)$. Besides, $\Omega$ is the set of all the Hermitian matrices and $\boldsymbol{\Lambda}_{i}=\operatorname{diag}\left(\lambda_{i}^{(1)}, \ldots, \lambda_{i}^{(M)}, 0, \ldots, 0\right)$.

\section{B. Optimal Offline Policy}

First we notice that optimization problem is a convex optimization problem, which can be proved by showing the objective function (13) to be concave and constraints linear. The detailed proof is similar to our previous work in [24] and is omitted here. Consequently, we can use convex optimization to solve it and the optimal solution satisfies the KKT conditions [25].

We define the Lagrangian function for any multipliers $\omega_{k} \geq$ $0, \mu_{k} \geq 0, \alpha_{k} \geq 0, \beta \geq 0, \gamma_{i j} \geq 0$ and $\eta \geq 0$ as

$$
\begin{aligned}
\mathcal{L}= & \sum_{i=1}^{N} \frac{T_{f}}{2} \log \left[\operatorname{det}\left(\mathbf{I}+\boldsymbol{\Lambda}_{i}^{\frac{1}{2}} \widetilde{\mathbf{Q}}_{i} \mathbf{\Lambda}_{i}^{\frac{1}{2}}\right)\right] \\
& -\sum_{k=1}^{N} \omega_{k}\left(\sum_{i=1}^{k} T_{f} p_{H, i}-\sum_{i=0}^{k-1} E_{i}\right) \\
& -\sum_{k=1}^{N-1} \mu_{k}\left(\sum_{i=0}^{k} E_{i}-\sum_{i=1}^{k} T_{f} p_{H, i}-E_{\max }\right) \\
& -\sum_{k=1}^{N} \alpha_{k}\left(p_{H, k}-\operatorname{tr}\left(\widetilde{\mathbf{Q}}_{k}\right)\right)+\sum_{k=1}^{N} \beta_{k} p_{H, k} \\
& -\eta\left(\frac{1}{N} \sum_{i=1}^{N}\left(\operatorname{tr}\left(\widetilde{\mathbf{Q}}_{i}\right)-p_{H, i}\right)-P_{G, \text { ave }}\right) \\
& +\sum_{i=1}^{N} \sum_{j=1}^{n_{t}} \gamma_{i j} \tilde{p}_{i}(j) .
\end{aligned}
$$

Additional complementary slackness conditions are as fol- 
lows

$$
\begin{aligned}
\omega_{k}\left(\sum_{i=1}^{k} T_{f} p_{H, i}-\sum_{i=0}^{k-1} E_{i}\right) & =0, \forall k, \\
\mu_{k}\left(\sum_{i=0}^{k} E_{i}-\sum_{i=1}^{k} T_{f} p_{H, i}-E_{\max }\right) & =0, k<N \\
\alpha_{k}\left(p_{H, k}-\operatorname{tr}\left(\widetilde{\mathbf{Q}}_{k}\right)\right) & =0, \forall k, \\
\beta_{k} p_{H, k} & =0, \forall k \\
\eta\left(\frac{1}{N} \sum_{i=1}^{N}\left(\operatorname{tr}\left(\widetilde{\mathbf{Q}}_{i}\right)-p_{H, i}\right)-P_{G, \text { ave }}\right) & =0, \\
\gamma_{i j} \tilde{p}_{i}(j) & =0, \forall i, j .
\end{aligned}
$$

Then we apply the KKT optimality conditions to the Lagrangian function (18). By using the fact that [26]

$$
\begin{aligned}
& \mathrm{d}\left\{\log \operatorname{det}\left[\mathbf{I}+\boldsymbol{\Lambda}^{\frac{1}{2}} \widetilde{\mathbf{Q}} \boldsymbol{\Lambda}^{\frac{1}{2}}\right]\right\} \\
= & \operatorname{tr}\left[\boldsymbol{\Lambda}^{\frac{1}{2}} \boldsymbol{\Psi}^{-1} \boldsymbol{\Lambda}^{\frac{1}{2}} \mathrm{~d} \widetilde{\mathbf{Q}}\right],
\end{aligned}
$$

where $\boldsymbol{\Psi}=\mathbf{I}+\boldsymbol{\Lambda}^{\frac{1}{2}} \widetilde{\mathbf{Q}} \boldsymbol{\Lambda}^{\frac{1}{2}}$, and setting $\partial \mathcal{L} / \partial \widetilde{\mathbf{Q}}_{i}=$ $\partial \mathcal{L} / \partial p_{H, i}=0$, we get the optimal power allocation as

$$
\begin{aligned}
\tilde{p}_{i}^{*}(j) & =\left[\frac{1}{\eta / N-\alpha_{i}}-\frac{1}{\lambda_{i}^{(j)}}\right]^{+}, \\
\text {or } \quad \tilde{p}_{i}^{*}(j) & =\left[\frac{1}{\sum_{k=i}^{N}\left(\omega_{k}-\mu_{k}\right)-\beta_{i}}-\frac{1}{\lambda_{i}^{(j)}}\right]^{+} .
\end{aligned}
$$

$\left\{\lambda_{i}^{(j)}, j=1, \ldots, M\right\}$ are the singular values of the channel matrix $\mathbf{H}_{i}$ as shown before and $[x]^{+}=\max \{x, 0\}$.

In addition, when solving this optimization problem, we find that $\widetilde{\mathbf{Q}}_{i}$ must be diagonal for the first $M$ columns with the diagonal element to be $\left\{\tilde{p}_{i}^{*}(j), j \leq M\right\}$, which means,

$$
\widetilde{\mathbf{Q}}_{i}=\operatorname{diag}\left(\tilde{p}_{i}{ }^{(1)}, \ldots, \tilde{p}_{i}^{*}(M), 0, \ldots, 0\right) .
$$

Based on expressions (26) and (27), we have following observations.

1) Different parallel channels in the same slot have the same water level. First, we fix the time dimension to see power allocation for parallel channels. On the right hand of Eqs. (26) and (27), the first part has no relation with the spatial item index, i.e., $j$. So the water level,

$$
\nu=1 /\left(\eta-\alpha_{i}\right)=1 /\left(\sum_{k=i}^{N}\left(\omega_{k}-\mu_{k}\right)-\beta_{i}\right),
$$

is constant for different antennas in the same time slot. The only difference lies on the second part, i.e., $1 /\left(\lambda_{i}^{(j)}\right)$, which is the singular value of the channel matrix. From this observation, we can see this optimal power allocation is the same as the traditional MIMO power allocation with power grid supply. Thus, the traditional water-filling [27] can be used to determine power allocation for different antennas in the same time slot.
2) While for the optimal power allocation in different slots, we consider it from two different kinds of conditions, where the power grid energy is non-zero or zero respectively.

2.a) The slots with non-zero grid power allocation achieve the same water level. If the power grid energy is non-zero, i.e, $p_{G, i}^{*}=p_{i}^{*}-p_{H, i}^{*}>0$,from the complementary slackness condition (21) we know that $\alpha_{i}=0$. Hence, the optimal allocation tries to achieve the constant water level $\nu=1 / \eta$, and the power is provided either by energy harvesting and power grid, or by power grid only $\left(p_{H, i}^{*}=0\right)$, which is

$$
\tilde{p}_{i}^{*}(j)=\left[\nu-\frac{1}{\lambda_{i}^{(j)}}\right]^{+} .
$$

Note that although the optimal power allocation depending on the constant water level is unique, there are more than one solution for $p_{H, i}^{*}$ and $p_{G, i}$ on condition that the constraints (5) and (6) are satisfied. This observation tells us that, no matter which slot and which antenna, if there is power grid energy allocated, the water level must be the same. This comes from the reason that the water level $\nu=1 / \eta$ is independent from both the spatial item $j$ and the temporal item $i$.

2.b) The optimal power allocation for slots with zero grid power can be achieved by the directional water-filling scheme. If the optimal power is supplied only by the harvested energy, i.e., $p_{i}^{*}=p_{H, i}^{*}$, the Lagrangian multiplier $\alpha_{i}$ can be any nonnegative value. Based on Eq. (27), the optimal water level is determined as

$$
\nu_{i}=\frac{1}{\sum_{k=i}^{N}\left(\omega_{k}-\mu_{k}\right)},
$$

followed by the optimal power allocation to be

$$
\tilde{p}_{i}^{*}(j)=\left[\nu_{i}-\frac{1}{\lambda_{i}^{(j)}}\right]^{+} .
$$

This result is exactly the optimal power allocation observed in our previous work [24]. Note that here we consider the power allocation for different slots and $\nu_{i}$ is the water level for slot $i$. We pick up the core idea called directional water-filling in the single antenna scenario [11]. There is a right permeable tap between two adjacent slots, which allows energy flow from left to right only. This comes from the reason that the harvested energy is causal. Further, the transferred energy can not be larger than $E_{\max }$ minus the harvested energy in the next slot due to the limited battery capacity.

Based on this, for the harvested energy powered only scenario, we present the main idea of our Spatial-Temporal Water-Filling $(S T-W F)$ policy. First, we use up all the energy harvested in each slot by traditional water-filling for different antennas to get an initial water level. Then, we compare the initial levels between two adjacent time slots, e.g., slot $j$ and slot $j+1$. If the water level in slot $j$ is higher than slot $j+1$, energy can flow from the previous slot to the latter one to achieve a constant water level. But the energy can not flow in the opposite direction because of the energy causality. In addition, the energy can not flow too much to make the latter slot overflow. In the duration of energy flows, the water 


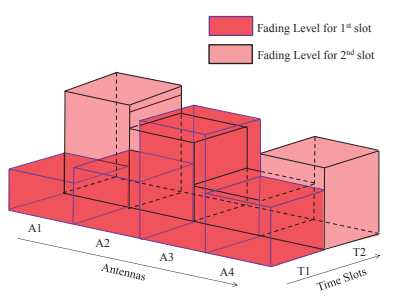

(a) Fading states

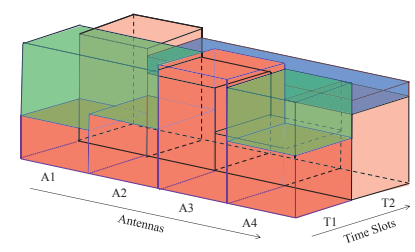

(d) Initial water level: Case 2

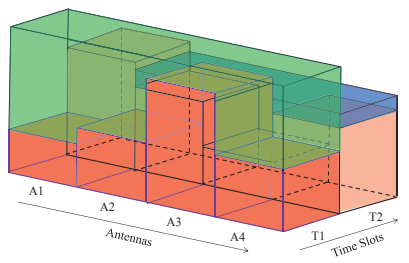

(g) Initial water level: Case 3

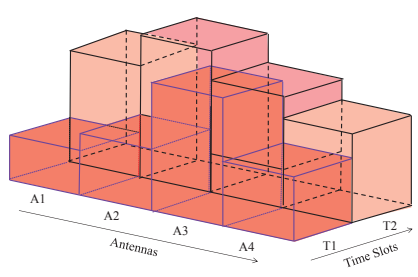

(j) Initial fading level: Case 4

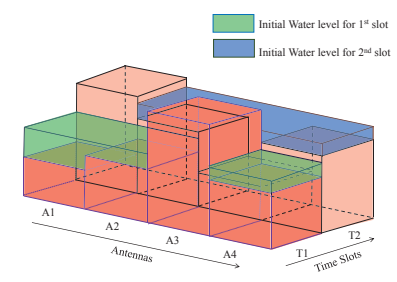

(b) Initial water level: Case 1

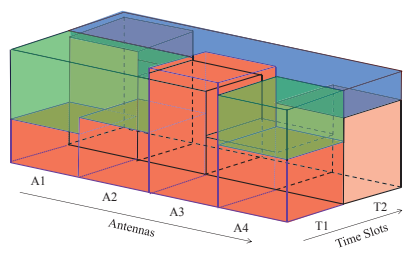

(e) ST-WF water level: Case 2

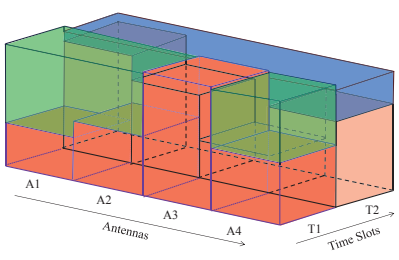

(h) ST-WF water level: Case 3

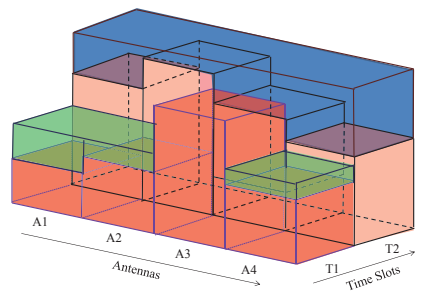

(k) ST-WF water level: Case 4

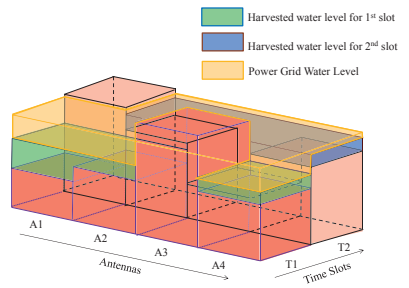

(c) Optimal water level: Case 1

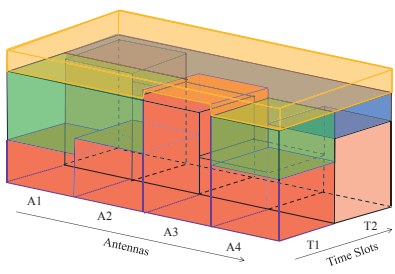

(f) Optimal water level: Case 2

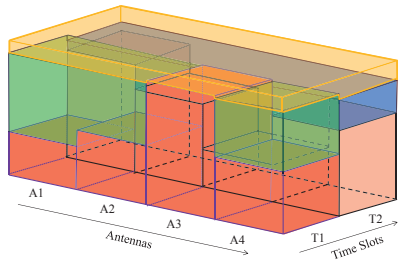

(i) Optimal water level: Case 3

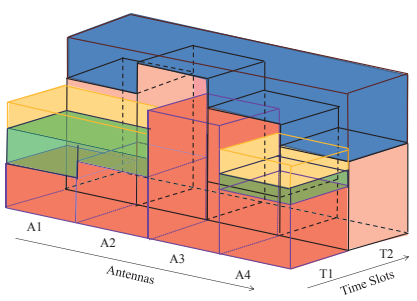

(1) Optimal water level: Case 4

Fig. 2. Examples for the 2-stage ST-WF operation.

level for different antennas in the same slot must stay the same. There is an important note that the new water level in each is not equal to its initial level plus the inflow water level minus the outflow water levels. This comes from the reason that different antennas in the same slot may have different kinds of fading levels, leading to unequal energy allocation when harvested energy inflows. In other words, the initial water level helps to decide whether there is energy to flow but can not directly decide the accurate optimal level. And that is the reason why the spatial domain and the temporal domain are not completely separate.

Having the above observations, we propose a simple optimal offline power allocation policy, namely two-stage spatialtemporal water-filling (two-stage ST-WF). Specifically, we divide the power allocation into two stages. In the first stage, the system allocates the harvested energy using spatial-temporal water-filling based on the original channel fading level. After allocating the harvested energy, we freeze the new water level, which becomes the new harvested water level for the second stage. And in the second stage, the traditional water-filling is performed to allocate the power grid energy on the frozen harvested water level. Then, the optimal power allocation can be obtained.

Actually, the harvested energy powered only scenario and the power grid only scenario are both special cases for the hybrid energy powered scenario. And the proposed two-stage spatial-temporal water-filling algorithm can be easily implemented for these two special cases. Specifically, as mentioned above the spatial temporal water-filling scheme is suitable for the harvested energy only scenario. And for the power grid only scenario, the first stage of using spatial-temporal waterfilling actually allocates zero harvested energy and the optimal power allocation is using conventional water-filling to allocate the power grid energy. 
For better understanding of the proposed two-stage spatialtemporal water-filling algorithm, examples are shown in Fig. 2 to give an intuitive explanation. We illustrate a total of 2 time slots . Both the transmitter and the receiver are equipped with 4 antennas. The fading level for the first three examples in each time slot and each channel is shown in Fig. 2(a), which is a three-dimension figure presenting both spatial and temporal fading. And the fading level for the forth example is shown in Fig. 2(j).

The first case is that the initial water level, which is set by using traditional water-filling (WF) for different antennas in the same slot, in the first slot is lower than that in the second slot, as shown in Fig. 2(b). Because of the energy causality, the optimal water level is the same as Fig. 2(b) after the STWF operation in the first stage since the water in the first slot can not flow to the second one. And then, traditional WF is used to allocate the power grid energy based on the frozen harvested water level to get the final optimal power condition in Fig. 2(c).

The second condition is a little higher initial water level in the first slot than the second one as Fig. 2(d). So the harvested energy can flow to the second slot to achieve a constant level as shown in Fig 2(e) and the harvested energy is frozen. Then also, after traditional WF for the power grid energy in the second stage, the optimal water level for these two kinds of power supplies is obtained as Fig. 2(f).

For the third example, we give the third example in Fig. 2(g)-2(i). In the first slot, there is much higher initial water level. As mentioned before, the harvested energy in the first slot can flow to the second one. But because of the battery capacity constraint, we can not allocate so much harvested energy to make the water levels the same. Thus, there is still a distance for the after the first stage as Fig 2(h). And Fig. 2(i) shows the finial optimal water level after the second stage to allocate the power grid energy.

Besides, we want to address that the final optimal water level is affected by the system parameters, such as energy arrivals and channel fading levels. In addition, the optimal water-level is not always the same for all time-slots and all parallel channels. We give the last example in Fig. 2(j)-2(1). The channel fading level in the second slot is higher than the first slot as Fig. 2(j). From Fig. 2(k), we can see that after the harvested energy allocation in the first stage, there is much higher water level in the second time slot. And the grid power is allocated only in the first slot and is not enough to make the optimal water levels the same as Fig. 2(1).

Note that the proposed two-stage ST-WF is just one of the optimal power allocation polices. For all the optimal allocation polices, they define the same unique optimal water level and the differences are the constitute of harvested energy and power grid energy for the total water level. For instance, if one optimal power for slot 1 and slot 2 is $p_{1}=p_{2}=2, p_{H, 1}=p_{H, 2}=1$, then the power allocation of $p_{1}=p_{2}=2, p_{H, 1}=0.5, p_{H, 2}=1.5$ is also optimal. In summary, the two-stage ST-WF gives the unique optimal total power allocation for each slot, but the proportion between the harvested energy and the power grid energy can be different.

The two-stage ST-WF algorithm are elucidated in the ap- pendix as Algorithm 1.

\section{Offline Power Grid Energy Minimization}

Now in this section, let us return to our original problem. We consider how to minimize the power grid energy based on the previous results. First we give a clear description about the power grid energy minimization problem. It is assumed that the there are $B$ bits to be transmitted within a given deadline, i.e., $N$ slots, using both the harvested energy and the power grid energy. Our objective is to minimize the power grid energy consumption under the constraints of energy harvesting causality, the limited battery capacity and the non-negative power allocation. In this section, we also analyze this problem in the offline setting and the online problem will be left in the next section. Using the same notations as the previous sections, we give the mathematic expression about this grid power minimization problem as follows,

$$
\begin{array}{ll}
\min & \sum_{i=1}^{N} T_{f}\left(p_{i}-p_{H, i}\right), \\
\text { s.t. } & (5),(6),(9), \text { and }(10), \\
& \sum_{i=1}^{N} \frac{T_{f}}{2} \log \left[\operatorname{det}\left(\mathbf{I}+\mathbf{H}_{i} \mathbf{Q}_{i} \mathbf{H}_{i}^{\mathrm{H}}\right)\right]=B .
\end{array}
$$

Actually, this problem can also be solved using Lagrangian function. But here, we use another way by showing that it is the dual problem of the throughput maximization problem to have a better understanding of the optimal structure.

Lemma 1. Given the average grid power, the maximum transmitted bits $B_{\max }=f\left(P_{G, a v e}\right)$ is a monotonic increasing function of $P_{G, \text { ave }} \geq 0$.

Intuitively, if the average grid power increases, we can first use the original grid power to transmit the same amount of bits and then use extra power to send more. Thus, the total number of transmitted bits must be more.

Proof: We set $P_{G \text {,ave, } 1}<P_{G \text {,ave } 2}$ and the maximum transmitted bits $B_{\max , 1}$ and $B_{\max , 2}$ respectively. Based on the previous section, we know that

$$
\begin{aligned}
B_{\max , k} & =\sum_{i=1}^{N} \frac{T_{f}}{2} \log \left[\operatorname{det}\left(\mathbf{I}+\mathbf{H}_{i, k} \mathbf{Q}_{i, k} \mathbf{H}_{i, k}^{\mathrm{H}}\right)\right] \\
& =\sum_{i=1}^{N} \frac{T_{f}}{2} \log \left[\operatorname{det}\left(\mathbf{I}+\boldsymbol{\Lambda}_{i, k} \widetilde{\mathbf{Q}}_{i, k} \mathbf{\Lambda}_{i, k}^{\mathrm{H}}\right)\right] \\
& =\sum_{i=1}^{N} \frac{T_{f}}{2} \log \left\{\prod_{j=1}^{M}\left[1+\lambda_{i, k}^{(j)} \tilde{p}_{i, k}^{*}(j)\right]\right\} \\
& =\sum_{i=1}^{N} \frac{T_{f}}{2} \sum_{j=1}^{M} \log \left\{\left[1+\lambda_{i, k}^{(j)} \tilde{p}_{i, k}^{*}(j)\right]\right\} \\
& \triangleq C\left(\tilde{p}_{i, k}^{*}(j), 1 \leq i \leq N, 1 \leq j \leq M\right)
\end{aligned}
$$

Then, because of the monotonicity of the log function and 
the optimal allocation of $B_{\max , 2}$, we have

$$
\begin{aligned}
B_{\max , 1}= & C\left(\tilde{p}_{i, 1}^{*}(j), 1 \leq i \leq N, 1 \leq j \leq M\right) \\
<\quad & C\left\{\tilde{p}_{i, 1}^{*}(j), 1 \leq i \leq N-1,1 \leq j \leq M\right. \\
& \tilde{p}_{N, 1}^{*}(j), 1 \leq j \leq M-1 \\
& \left.\tilde{p}_{N, 1}^{*}(M)+N\left(P_{G, \text { ave }, 2}-P_{G, \text { ave }, 1}\right)\right\} \\
\leq & C\left(\tilde{p}_{i, 2}^{*}(j), 1 \leq i \leq N, 1 \leq j \leq M\right)
\end{aligned}
$$

In addition, it is clear that $B_{\max }=f\left(P_{G \text {,ave }}\right)$ is continuous because of the continuity of $\log$ function.

Note that $B_{0}=f(0)>0$, which means that when there is no power grid energy, we can still transmit some bits as long as the harvested energy is non-zero for all slots. And $B_{0}$ is related with the energy harvesting process.

Based on the Lemma 1, the following proposition about power grid energy minimization is obtained.

Proposition 1. Given the number of bits $B$ to be transmitted using the hybrid energy harvesting and power gird supplies, and given the harvested energy arrivals, which means the offline condition, the minimum required power grid energy can be shown as,

$$
P_{G, \min }= \begin{cases}f^{-1}(B), & B>B_{0} \\ 0, & B \leq B_{0}\end{cases}
$$

where $B_{0}$ is the maximum bits that can be transmitted by the harvested energy only.

Note that here we consider the offline condition and $B_{0}$ is fixed given the harvested energy arrivals.

0 .

Proof: First if $B$ is less than $B_{0}$, it is clear that $P_{G, \min }=$

Otherwise if the total waiting bits are larger than $B_{0}$, we set $B=f(P)$. Based on Lemma 1, we first know that the inverse function of $f$ exists, which is denoted as $f^{-1}$. If $P_{G, \min }>P$, we have $\widetilde{B}=f\left(P_{G, \min }\right)>f(P)=B$, which means we can get higher throughput using $P_{G \text {,min }}$ grid power. And this contradicts our assumption. The same contradiction holds for $P_{G, \text { min }}<P$. Thus, we have $P=P_{G, \text { min }}$, in other words, $B=f\left(P_{G, \min }\right)$. Then we operate $f^{-1}$ on both sides of the equation to get $f^{-1}\left[f\left(P_{G, \min }\right)\right]=P_{G, \min }=f^{-1}(B)$, which is the conclusion as elucidated in the first part of Eq. (37).

Proposition 1 tells us that when the number of bits to be transferred is less than or equal to $B_{0}$, the minimum grid power consumption is zero. Otherwise, we can first get the function $B=f(P)$ by using the proposed two-stage ST$\mathrm{WF}$ and then find the corresponding point on the curve to known the minimum grid power. As for finding the optimal power allocation, We give the detailed steps as Algorithm 2 in the appendix. We first use the ST-WF algorithm by setting $P_{G \text {,ave }}=0$ to get the optimal harvested energy allocation, which is also the optimal power allocation if the desired bits are less than $B_{0}$. On the other hand, if the desired bits are larger than $B_{0}$, we operate the orthodox WF to find the optimal power grid energy needed to cover the extra bits, which is step 6 in our algorithm.

\section{Online Power Grid EnERGy Minimization}

Until now, we still focus on the problem based on the offline condition with non-causal full information and get some interesting results. In this section, we will study the online power allocation scheduling under the online condition with causal information. We only have the knowledge of the current and past channel fading information and energy arrivals. Remember that our goal is to minimize the power grid energy consumption while transmitting a total of $B$ bits in $N$ time slots.

\section{A. Optimal Online Solution}

Here, we assume that both the energy arrival and the channel fading take independent values in different slots with probability density $f(E)$ and $f(\gamma)$ correspondingly. We model the system state slot $i$ as the remaining battery energy $e_{i}$ at the beginning of slot $i$ and the already transmitted number of bits $b_{i}$, which has the Markov property. Hence the the optimal online solution can be achieved by the MDP algorithm [22]. Besides the system state, the MDP algorithm requires another two key components, i.e., the action and the cost function. In our problem, the action is defined as the number of bits $\Delta b_{i}$ to be transmitted and the grid power $p_{G, i}$ in the current slot. Note that the total energy consumption $p_{i}$ can be determined by traditional water filling once $\Delta b_{i}$ and the channel states $\left\{\lambda_{i}^{(j)}\right\}$ are given. The per-stage function is the power grid energy consumption $p_{G, i}$. We impose an infinite cost if the transmitted bits are less than $B$ after the deadline. Then the cost-to-go function can be defined as

$$
\begin{aligned}
J_{N}\left(e_{N}, b_{N}\right) & =\left\{\begin{array}{l}
0, \quad b_{N} \geq B \\
+\infty, b_{N}<B
\end{array}\right. \\
J_{i}\left(e_{i}, b_{i}\right) & \left.=\mathbb{E}\left\{\min _{\Delta b_{i}, p_{G, i}}\left[p_{G, i}+J_{i+1}\left(e_{i+1}, b_{i+1}\right)\right]\right\} 39\right)
\end{aligned}
$$

where $e_{i+1}=\max \left\{e_{i}-\left[\left(p_{i}-p_{G, i}\right)\right]^{+}+E_{i}, E_{\max }\right\}, b_{i+1}=$ $b_{i}+\Delta b_{i}$ and $\mathbb{E}$ denotes the expectation for all possible channel states and energy arrivals.

Here the cost-to-go function $J_{i}\left(e_{i}, b_{i}\right)$ actually means the minimum expected grid power cost to finish the transmission from the $i$ th slot to the $N$ th slot given the battery level $e_{i}$ and the already transmitted bits $b_{i}$.

Since the total $B$ bits must be transmitted before the deadline, we give infinite grid power cost if the already transmitted bits $b_{N}$ in the $N$ th slot is less than $B$, which means that this bits outage condition is prohibited. On the other hand, if the total $B$ bits have been transmitted, the gird power cost or the cost-to-go function in the $N$ th slot is zero. That is reason why we choose the cost-to-go function in slot $N$ as eq. (38).

For the cost-to-go function in other slots except the last one, if the grid power cost $J_{i+1}\left(e_{i+1}, b_{i+1}\right)$ in slot $i+1$ is known, we can use eq. (39) to calculate that of slot $i$, which is the power grid cost $p_{G, i}$ in slot $i$ plus $J_{i+1}$. Here $\mathbb{E}$ in Eq. (38) means the expectation for all possible energy arrivals and channel conditions. We record the grid power cost $p_{G, i}$ and the transmitted bits $\Delta b_{i}$ in every slot to achieve the minimum expectation. Hence, from the slot $N$ to the first slot, the minimum grid power cost can be calculated recursively.

In addition for the MDP algorithm itself, the system parameters such as energy levels and transmitted bits are all 
quantized so that the cost-to-go function between different system states can be calculated. And the quantization is also cause of the curse of dimensionality, which leads to high calculation complexity.

In summary, we can calculate the cost function recursively from the last slot to the very beginning slot. The transmitter records all these cost-to-go functions and corresponding $\Delta b_{i}$ and $p_{G, i}$ as a look-up table. When the look-up table for all the quantized bits and energy levels is built, we can find the right power in this table from the first slot to the last one according the system states. Specifically, in each slot, the transmitter can decide the optimal power allocation based on the lookup table by sensing the channel states $\left\{\lambda_{i}^{(j)}\right\}$, the remaining battery energy $e_{i}$, the already transmitted bits $b_{i}$ and newly harvested energy $E_{i}$. The traditional water-filling is used to allocate energy to every antenna once the optimal power in a given slot is determined. In short, this MDP algorithm consists of two parts, namely building the look-up table from the last slot the first one and finding the right power allocation in the look-up table from the first slot to the end.

\section{B. Online Near-Optimal Solutions}

Though the MDP algorithm can get the optimal online result, its high complexity is also obvious due to the curse of dimensionality. Thus, in this section, we will propose some near-optimal algorithms based on the structure of the offline solution of Section III and Section IV. We assume that the MIMO channel follows independent fading probability density $f(\gamma)$.

1) Constant Water Level Algorithm. Recall that the optimal offline solution tries to achieve a constant water level. We can propose this constant water level algorithm, which is assumed that there is a constant water level $1 / \gamma_{0}$ for all the slots except for the slot $N$. The fixed water level can be calculated by solving the following equation

$$
\int_{\gamma_{0}}^{\infty} \frac{1}{2} \log \left(\frac{\gamma}{\gamma_{0}}\right) f(\gamma) \mathrm{d} \gamma=\frac{B}{N T_{f} M},
$$

and the power allocation for each antenna in each slot is calculated as

$$
p_{i}^{\text {Const }}(j)=\left[\frac{1}{\gamma_{0}}-\frac{1}{\lambda_{i}^{(j)}}\right]^{+} .
$$

Note that the water level $\gamma_{0}$ is not a pre-defined parameter but determined by solving eq. (40). Given the channel fading probability density $f(\gamma)$, total bits $B$, total number of slots $N$, the slot length $T_{f}$ and the number of parallel channels $M$, the unknown variable in eq. (40) is only $\gamma_{0}$. Hence we can use numerical method to solve eq. (40) to get the value of $\gamma_{0}$.

We given some explanations for eq. (40) in detail. In fact, both sides of eq. (40) denote the expected transmitted bits in every slot and every parallel channel. For eq. (40), the water level is given by $\frac{1}{\gamma_{0}}$. If the channel fading level is $\gamma$, the power allocated in this slot is $\left[\frac{1}{\gamma_{0}}-\frac{1}{\gamma}\right]^{+}$based on the water-filling scheme. According to Shannon Equation, $\frac{T_{f}}{2} \log \left(\frac{\gamma}{\gamma_{0}}\right)$ bits are transmitted. Then the expected transmitted bits can be obtained by taking integral for all possible $\gamma \geq \gamma_{0}$ with distribution function $f(\gamma)$.. While for the right side of eq. (40), $\frac{B}{N M}$ also denotes the average bits transmitted for each slot. Hence we have eq. (40).

In case of battery overflow, the harvested energy is used with higher priority. In other words, if the remaining harvested energy is enough to support $\sum_{j=1}^{M} p_{i}^{\text {Const }}(j) T_{f}$, we will use the harvested energy only. Then the power allocation and the battery condition can be calculated as (42)-(45).

$$
\begin{array}{r}
p_{i}=\sum_{j=1}^{M} p_{i}^{\text {Const }}(j), \\
p_{c, i}=e_{i-1}+E_{i-1}-p_{i}, \\
e_{i}=\max \left\{0, p_{c, i}\right\}, \\
p_{G, i}=\max \left\{0,-p_{c, i}\right\},
\end{array}
$$

where $e_{i}$ is the battery energy at the beginning of slot $i$.

2) Bits Aware Water Level Algorithm. Due to the variation of the channel gains, the constant water level may be not satisfying in the finite time transmission. So the second idea is adapting the water level in each slot based on the remaining bits to improve the performance. And the water level is decided by

$$
\int_{\gamma_{0, i}}^{\infty} \frac{1}{2} \log \left(\frac{\gamma}{\gamma_{0, i}}\right) f(\gamma) \mathrm{d} \gamma=\frac{B_{i}}{(N-i+1) T_{f} M},
$$

where $B_{i}$ denotes the remaining bits to be transmitted in slot $i$. The total power allocation for antennas, the power grid energy and the battery energy is calculated similarly as Eqs. (42) (45).

In addition, the parameter $\gamma_{0, i}$ is decided by solving eq. (46). And the explanation for why we choose eq. (46) is similar to the constant water level algorithm. The only difference is that the average transmitted bits is adjusted slot by slot for the right hand side of eq. (46).

Besides, the battery overflow protection is applied in both the constant water level algorithm and the bit-aware water level algorithm, which means that if $e_{i}+E_{\text {ave }}>E_{\max }$, the energy of $e_{i}+E_{\text {ave }}-E_{\max }$ must be used in the current slot. Here $E_{\text {ave }}$ denotes the average harvested energy for every slot. The complete procedure for these two algorithms are shown as Algorithm 3 in the appendix.

\section{NumERICAL RESUlTS}

In this section, we test the performance of the proposed algorithms. We use the Rayleigh fading channel with the average channel gain to be $0 \mathrm{~dB}$ and generate independent random matrices for each run. There are total 80 time slots and the MIMO system is equipped with 4 transmitting antennas and 4 receiving antennas. A total of 1000 monte carlo simulation runs are performed for different parameter setups. The harvested energy follows non-negative uniform distribution with mean $P_{H \text {,ave }}=E_{\text {ave }} / T_{f}=20 \mathrm{dBm}$ and is independent in different slots. And we set $T_{f}=1 \mathrm{~s}$. The initial battery level is set to be zero, which means there is no harvested energy at the beginning. While for $\gamma_{0}$ or $\gamma_{0, i}$, it is calculated by solving eq. (40) and eq. (46). For the values 


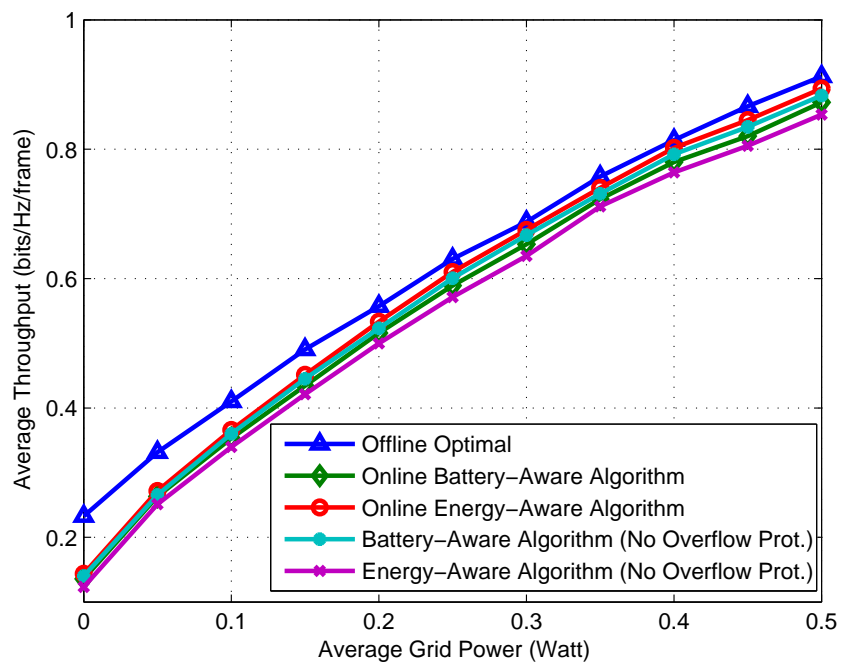

Fig. 3. Performance of average throughput versus average grid power in throughput maximization. $\left(N=80, P_{H, \text { ave }}=20 \mathrm{dBm}\right.$ and $\left.E_{\max }=0.3 \mathrm{~J}\right)$

of grid power, in the throughput maximization part, it is a variable in Fig. 3 as $\mathrm{x}$-axis. In the grid power minimization part, the values of grid power is the objective function we want to minimize as y-axis in Figs. 4-6.

\section{A. Throughput Maximization}

First we exam the performance of the throughput maximization problem discussed in Section III. We compare the optimal two-stage ST-WF with two online heuristic algorithms, namely the battery-aware water level algorithm and the energyaware water level algorithm. The idea for designing these two algorithms comes from the dual property of the throughput maximization problem and the power grid energy minimization problem. The difference is that the parameter to determine the water level is the given power supply instead of the number of packets as in the pervious section.

The battery-aware water level is calculated as

$$
\int_{\gamma_{0}}^{\infty}\left(\frac{1}{\gamma_{0}}-\frac{1}{\gamma}\right) f(\gamma) \mathrm{d} \gamma=\frac{P_{\mathrm{H}, \text { ave }}+P_{\mathrm{G}, \text { ave }}}{M} .
$$

The power allocation for each antenna is the same as (41). The energy-aware water level is decided by solving

$\int_{\gamma_{0, i}}^{\infty}\left(\frac{1}{\gamma_{0, i}}-\frac{1}{\gamma}\right) f(\gamma) \mathrm{d} \gamma=\frac{(N-i) E_{\text {ave }}+e_{i}}{(N-i+1) T_{f} M}+\frac{P_{G, \text { ave }}}{M}$

In addition, to avoid the risk of battery overflow, the battery overflow protection is also used as mentioned before. Actually, these two algorithms are very similar with those in ref. [11], where their algorithms are designed for the SISO scenario with only energy harvesting powered. We extend them into the MIMO condition by using traditional water-filling to allocate power among different antennas in the same slot. In addition, there is an important note that our algorithms consider the battery overflow protection while ref. [11] did not. And we use these algorithms without overflow protection for comparation.

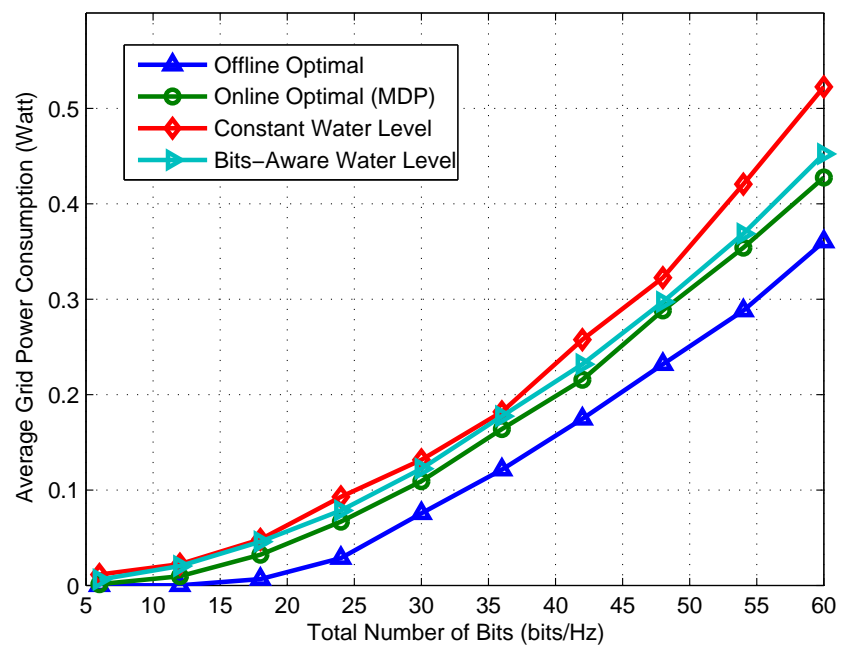

Fig. 4. Performance of average grid power consumption versus number of bits per bandwidth. $\left(N=80, P_{H \text {,ave }}=20 \mathrm{dBm}\right.$ and $\left.E_{\max }=0.3 \mathrm{~J}\right)$

The average throughput versus the average grid power is shown in Fig. 3. It shows that the energy-aware water level algorithm outperforms the battery-aware one because the energy-aware algorithm is adaptive to the energy condition. In addition, both of them approach the offline optimal solution as the average grid power increases, which is reasonable because the effect of harvested energy will gradually decrease while the effect of the power grid energy is the same for all of them. Besides, since our algorithms consider the finite battery capacity constraint and protect the battery overflow, we can see that the corresponding algorithms perform better.

\section{B. Power Grid Energy Minimization}

This part gives the numerical result of the power grid energy minimization problem based on the discussion of Section IV and Section V. We evaluate the performances of the optimal offline solution, optimal online solution (MDP) and proposed near-optimal online algorithms.

Fig. 4 shows the average grid power consumption achieved versus number of bits per bandwidth. From the numerical results, we can see that the proposed bit-aware water level algorithm performs better than the constant water level algorithm and is close to the optimal online solution given by MDP. This is because that the bit-aware adapts to the system conditions and can better utilize the energy. In the low bits region, different algorithms achieve the similar performance since the power grid energy consumption will all turn to zero. Besides, the average grid power consumption grows exponentially with the total number of bits, which corresponds with the Shannon equation.

We then evaluate the numerical performance results of the algorithms under different battery capacities as shown in Fig. 5. The number of bits per bandwidth is $30 \mathrm{bits} / \mathrm{Hz}$. With higher battery capacity, the average grid power consumption will decrease. Besides, the result shows that when the battery capacity is larger than a threshold, the power allocation 


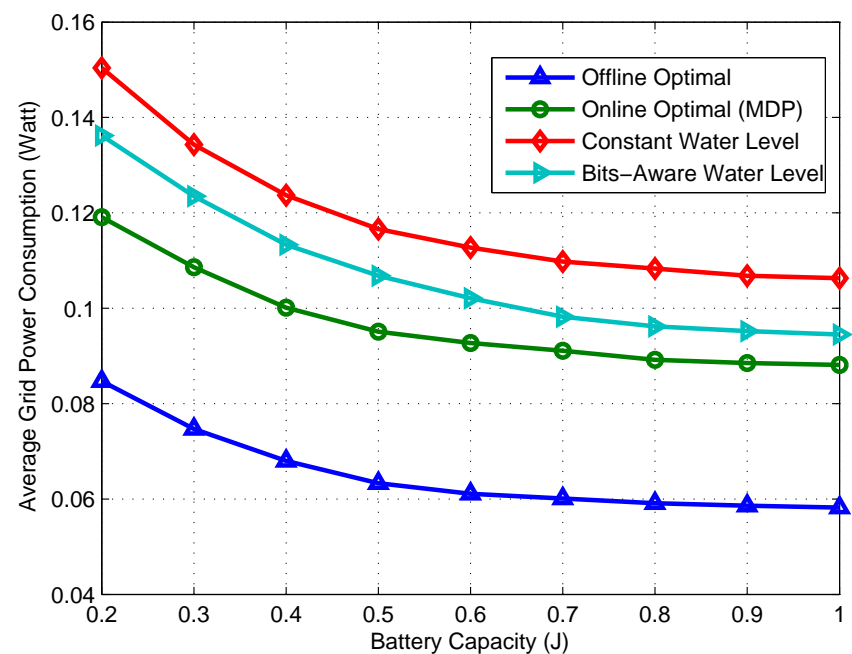

Fig. 5. Performance of average grid power consumption versus battery capacity. $\left(N=80, P_{H \text {,ave }}=20 \mathrm{dBm}\right.$ and the number of bits per bandwidth is $30 \mathrm{bits} / \mathrm{Hz}$ )

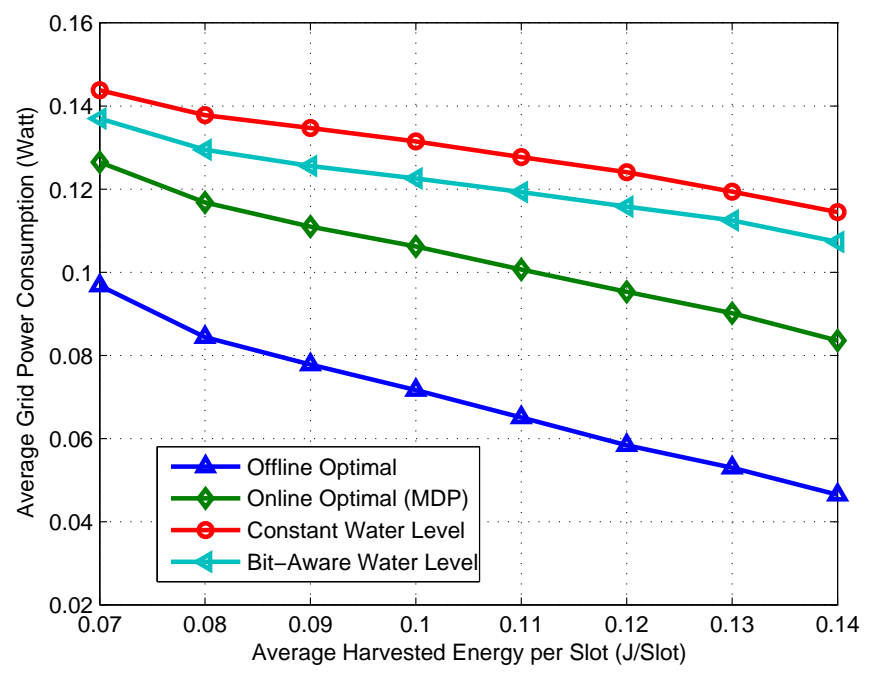

Fig. 6. Performance of average grid power consumption versus the average harvested energy. $\left(N=80, E_{\max }=0.3 \mathrm{~J}\right.$ and the number of bits per bandwidth is $30 \mathrm{bits} / \mathrm{Hz}$ )

becomes almost constant. This comes from the reason that the capacity constraint is easy to be satisfied and then has no influence on the final results. We define the relative battery capacity as $M_{B}=E_{\max } /\left(P_{H, \text { ave }} T_{f}\right)$. We can see that when $M_{B}<6$, the average grid power consumption decrease with higher battery capacity and when $M_{B} \geq 6$, the average grid power consumption will be constant. So, we can set the relative battery capacity to be 6 to achieve a satisfying performance for real application. Also, the bit-aware water level algorithm performs better than the constant water level algorithm, which is similar to Fig. 4.

The last performance is about the average grid power consumption versus the average harvested energy as shown in Fig. 6. The number of bits per bandwidth is also 30
bits/Hz. Again, the bit-aware water level algorithm is better. Note that the distance between the proposed the near-optimal algorithms and the optimal online algorithm increases when the average harvested energy is higher. This results from the fact that we use more harvested energy in the high average harvested energy region, which is harder to be predicted compared to the power grid energy. Another note is that the average grid power consumption decreases almost linear with the average harvested energy. Under the given parameters, one watt increase of the harvested energy per slot can decrease 0.4 watt average grid power consumption for the constant water level algorithm and the bit-aware water level algorithm, 0.5 watt for the MDP algorithm and 0.6 watt for the optimal offline policy.

Based on Figs. 4-6, we can see that the offline algorithm performs best since it has more information than the online policy. For online conditions, the MDP algorithm needs less grid power and performs better than the other two near-optimal online algorithms, which shows that the MDP algorithm is indeed the optimal online solution.

\section{CONCLUSION}

In this paper, we have analyzed the power grid energy minimization problem in the MIMO system with hybrid energy harvesting and power gird supplies. We first solve the throughput maximization problem and propose the optimal solution called two-stage spatial-temporal water-filling algorithm under the offline condition with finite battery capacity. We then solve the offline power grid energy minimization problem by using its dual property with the throughput maximization problem. For the online condition, we give the optimal policy by using MDP. In addition, motivated by the offline solution, online near-optimal algorithms with reduced complexity, namely the constant water level algorithm and the bit-aware water level algorithm, are also proposed and evaluated by numerical simulations. Numerical results show that the bit-aware water level algorithm outperforms the constant one and is close to the optimal MDP online results. Besides, the results tell us that the average gird power consumption decreases with battery capacity and turns to be constant if the battery capacity is larger than a threshold. And the trade-off between the average harvested energy and the average grid power consumption is almost linear from the numerical results.

\section{APPENDIX}

\section{APPENDIX A}

\section{OPTIMAL OFFLINE THROUGHPUT MAXIMIZATION} ALGORITHM

\footnotetext{
Algorithm 1: The Two-Stage Spatial-Temporal Water-Filling Input:

Number of slots: $N$; Number of parallel channels: $M$; Singular values of $\mathbf{H}_{i}:\left\{\lambda_{i}^{(j)}, i=1, \ldots, N, j=1, \ldots, M\right\}$; Battery capacity $E_{\text {max }}$

Harvested energy for each slot $E_{i} \leq E_{\text {max }}$;

Average power grid energy $P_{G \text {,ave }}$.
}

\section{Output:}


Optimal power allocation $\tilde{p}_{i}^{*}(j), \tilde{p}_{H, i}^{*}(j)$.

Maximum throughput $B_{\max }$

for $i=1: N$ do

Find $v_{i}^{\prime}$ so that

$$
\sum_{j=1}^{M} T_{f} p_{i}(j)=E_{i}, p_{i}(j)=\left[v_{i}^{\prime}-\frac{1}{\lambda_{i}^{(j)}}\right]^{+} .
$$

Find $v_{i, \max }$ so that

$$
\sum_{j=1}^{M} T_{f} p_{i}(j)=E_{\max }, p_{i}(j)=\left[v_{i}^{\prime}-\frac{1}{\lambda_{i}^{(j)}}\right]^{+} .
$$

end for

Set $\Phi=\emptyset$.

for $i=N-1:-1: 1$ do

if $v_{i}^{\prime} \leq v_{i+1}^{\prime}$ then

Continue.

else

while There is $v_{k}^{\prime}<v_{i}^{\prime}, k>i, k \notin \Phi$. do

Find $k_{\max }=\max \left\{k \mid v_{k}^{\prime}<v_{i}^{\prime}, k>i, k \notin \Phi\right\}$.

Set $\Gamma_{i}=\left\{i<k \leq k_{\max }, k \notin \Phi\right\}$

Update $v_{l}^{\prime}=v^{\prime}, l \in \Gamma_{i}$ such that

$$
\sum_{l \in \Gamma_{i}} \sum_{j=1}^{M} T_{f} p_{l}(j)=\sum_{l=i}^{k_{\max }} E_{l}-N_{k, i} E_{\max }
$$

and

$$
p_{l}(j)=\left[v^{\prime}-\frac{1}{\lambda_{l}^{(j)}}\right]^{+},
$$

where $N_{k, i}=k_{\max }-i+1-\left|\Gamma_{i}\right|$ and $\left|\Gamma_{i}\right|$ is the number of elements in set $\Gamma_{i}$.

Find the set $\Theta$ where $v_{n}^{\prime}>v_{n, \max }^{\prime}, n \in \Theta$.

Update $v_{n}^{\prime}=v_{n, \max }^{\prime}$, where $n \in \Theta$.

Update $\Gamma_{i}=\Gamma_{i}-\Theta$ and $\Phi=\Phi \cup \Theta$.

\section{end while}

end if

end for

Find $v$ such that

$$
\sum_{i=1}^{N} \sum_{j=1}^{M} p_{G, i}(j)=N P_{G, \text { ave }},
$$

and

$$
p_{G, i}(j)=\left[v-\max \left(\frac{1}{\lambda_{i}^{(j)}}, v_{i}^{\prime}\right)\right] .
$$

return $\tilde{p}_{H, i}^{*}(j)=\left[v_{i}^{\prime}-\frac{1}{\lambda_{i}^{(j)}}\right]^{+}$and $\tilde{p}_{i}^{*}(j)=$ $\left[v-\frac{1}{\lambda_{i}^{(j)}}\right]^{+}$.

return $B_{\max }=\sum_{i=1}^{N} \frac{T_{f}}{2} \sum_{j=1}^{M} \log \left[1+\lambda_{i}^{(j)} p_{i}^{*}(j)\right]$

\section{APPENDIX B}

Optimal OfFline Power Grid Energy Minimization

\section{ALGORITHM}

\section{Algorithm 2: Offline Power Grid Energy Minization}

Input:

Number of slots: $N$; Number of parallel channels: $M$;

Singular values of $\mathbf{H}_{i}:\left\{\lambda_{i}^{(j)}, i=1, \ldots, N, j=1, \ldots, M\right\}$;

Battery capacity $E_{\max }$;

Harvested energy for each slot $E_{i} \leq E_{\max }$;

Total bits to be transferred $B$.

\section{Output:}

Optimal power allocation $\tilde{p}_{i}^{*}(j), \tilde{p}_{H, i}^{*}(j)$.

Minimum power grid energy consumption $p_{G, \min }$

Set $P_{G \text {,ave }}=0$.

Find the maximum throughput $B_{0}$ and optimal harvested energy allocation $p_{H, i}^{*}(j)$ by using Algorithm 1 when $P_{G, \text { ave }}=0$.

if $B \leq B_{0}$ then

return $p_{G, \min }=0$ and $\tilde{p}_{i}^{*}(j)=\tilde{p}_{H, i}^{*}(j)$.

else

Find $v_{G}$ such that

$$
\sum_{i=1}^{N} \frac{T_{f}}{2} \sum_{j=1}^{M} \log \left[1+\lambda_{i}^{(j)}\left(\tilde{p}_{G, i}^{*}(j)+\tilde{p}_{H, i}^{*}(j)\right)\right]=B,
$$

and

$$
\tilde{p}_{G, i}^{*}(j)=\left[v_{G}-\left(\frac{1}{\lambda_{i}^{(j)}}+\tilde{p}_{H, i}^{*}(j)\right)\right]^{+}
$$

return

$$
p_{G, \min }=\frac{1}{N} \sum_{i=1}^{N} \sum_{j=1}^{M} \tilde{p}_{G, i}^{*}(j)
$$

and

$$
\tilde{p}_{i}^{*}(j)=\tilde{p}_{G, i}^{*}(j)+\tilde{p}_{H, i}^{*}(j)
$$

end if

\section{APPENDIX C}

OnLine Sub-OPTIMAl POWER GRID ENERgY MINIMIZATION ALGORITHM

\section{Algorithm 3: Online Power Grid Energy Minization}

\section{Input:}

Number of slots: $N$; Number of parallel channels: $M$;

Singular values of $\mathbf{H}_{i}:\left\{\lambda_{i}^{(j)}, i=1, \ldots, N, j=1, \ldots, M\right\}$;

Battery capacity $E_{\text {max }}$; Harvested energy per slot $E_{\text {ave }}$

Harvested energy for each slot $E_{i} \leq E_{\max }$;

Total bits to be transferred $B$.

\section{Output:}

Power allocation $\tilde{p}_{i}^{*}(j), \tilde{p}_{H, i}^{*}(j)$.

1: for $i=1: N$ do

2: $\quad$ Calculate the water level $\gamma_{i}$ using Eq. (40) or Eq. (46).

3: $\quad$ Set $p_{\mathrm{S}}=\sum_{j=1}^{M}\left[\frac{1}{\gamma_{i}}-\frac{1}{\lambda_{i}^{(j)}}\right]$.

4: $\quad$ if $p_{\mathrm{S}} T_{f} \geq e_{i-1}+E_{i-1}$ then 


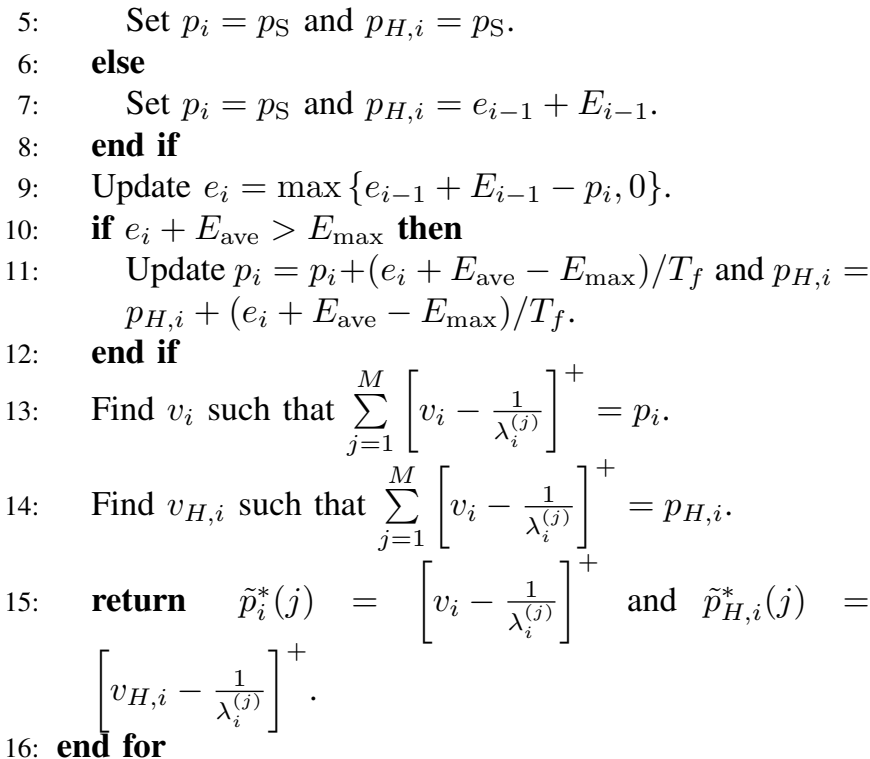

16: end for

[17] Y. Cui, K. N. Lau, and Y. Wu, "Delay-aware BS discontinuous transmission control and user scheduling for energy harvesting downlink coordinated MIMO systems", IEEE Trans. Signal Processing, Vol. 60, No. 7, Jul. 2012.

[18] T. Han, N. Ansari, "On Optimizaing Green Energy Utilization for Cellular Networks with Hybrid Energy Supplies", IEEE Trans. Wireless Commun., Vol. 12, No. 8, August 2013.

[19] J. Gong, S. Zhou, Z. Niu, "Optimal power allocation for energy harvesting and power grid coexisting wireless communication systems", IEEE Trans. Commun., Vol. 61, No. 7, Jul. 2013.

[20] Y. Liu, Introduction to the Internet of Things, Science Press, Beijing, China, 2010.

[21] T. L. Marzetta, "Noncooperative cellular wireless with unlimited numbers of base station antennas", IEEE Trans. Wireless Commun., Vol. 9, No. 11, Nov. 2010

[22] D. P. Bertsekas, "Dynamic programming and optimal control", 3rd ed., Athena Scientific, Belmont, Massachusetts, 2005.

[23] E. Telatar, "Capacity of multi-antenna Gaussian channels", AT\&T-Bell Labs Internal Tech. Memo., June 1995.

[24] C. Hu, J. Gong, X. Wang, S. Zhou and Z. Niu, "Spatial-Temporal WaterFilling power allocation for MIMO systems with harvested energy", IEEE ICCC, Aug. 2013.

[25] S. Boyd and L. Vandenberghe, Convex optimization, Cambridge University, 2004

[26] X. Zhang, Matrix Analysis and Applications, Tsinghua University Press, Beijing, 2004

[27] A. Goldsmith, Wireless Communications, Cambridge University, 2005.

\section{REFERENCES}

[1] C. Han, et al., "Green radio: radio techniques to enable energy-efficient wireless networks", IEEE Commun. Mag., Vol. 49, No. 6, June 2011.

[2] S. Zhou, J. Gong, Z. Yang, Z. Niu, P. Yang, "Green Mobile Access Network with Dynamic Base Station Energy Saving", ACM MobiCom. Vol. 9. No. 262, 2009.

[3] "Sustainable energy use in mobile communications", Ericsson Inc., white paper, Aug. 2007.

[4] E. Oh, B. Krishnamachari, X. Liu, Z. Niu, "Toward dynamic energyefficient operation of cellular network infrastructure", IEEE Commun. Mag., Jun. 2011

[5] D. Valerdi, Q. Zhu, K. Exadaktylos, S. Xia, M. Arranz, R. Liu, and D. Xu, "Intelligent energy managed service for green base stations", IEEE Globecom GreenComm. Workshop, Dec. 2010

[6] M. Gorlatova, A. Wallwater, and G. Zussman, "Networking low-power energy harvesting devices: measurements and algorithms", IEEE International Conference on Computer Communications (INFOCOM), Shanghai, April 2011.

[7] M. Gatzianas, L. Georgiadis, and L. Tassiulas, "Control of wireless networks with rechargeable batteries", IEEE Trans. Commun., Vol. 9, No. 2, Feb. 2010

[8] V. Sharma, U. Mukherji, V. Joseph, and S. Gupta, "Optimal energy management policies for energy harvesting sensor nodes", IEEE Trans. Wireless Commun., Vol. 9, No. 4, Apr. 2010

[9] C. K. Ho and R. Zhang, "Optimal energy allocation for wireless communications powered by energy harvesters", Int. Symposium Inf. Theory, Austin, Texas, U.S.A., Jun. 13-18, 2010

[10] K. Tutuncuoglu and A. Yener, "Optimum transmission policies for battery limited energy harvesting nodes", IEEE Trans. Commun., Vol. 11, No. 3, Mar. 2012

[11] O. Ozel, K. Tutuncuoglu, J. Yang, S. Ulukus, and A. Yener, "Transmission with energy harvesting nodes in fading wireless channels: optimal policies", IEEE J. Selected Area Commun., Vol. 29, No. 8, Sept. 2011

[12] J. Yang, O. Ozel, and S. Ulukus, "Broadcasting with an energy harvesting rechargeable transmitter", IEEE Trans. on Wireless Commun., Vol. 11, Issue 2, pp. 571-583, February 2012.

[13] J. Yang and S. Ulukus, "Optimal packet scheduling in a multiple access channel with rechargeable nodes", IEEE ICC, Kyoto, June 2011.

[14] E. U. Biyikoglu, B. Prabhakar, and A. El Gamal, "Energy-efficient transmission over a wireless link via lazy packet scheduling", IEEE/ACM Trans. Networking, Vol. 10, No. 4, pp. 487-499, Aug. 2002

[15] W. Chen, M. J. Neely, and U. Mitra, "Energy efficient scheduling with individual packet delay constraints: offline and online results", IEEE Infocom, 2007

[16] M. A. Zafer and E. Modiano, "A calculus approach to energy-efficient data transmission with quality-of-service constraints", IEEE/ACM Trans. Networking, Vol. 17, No. 3, pp. 898-911, Jun. 2009

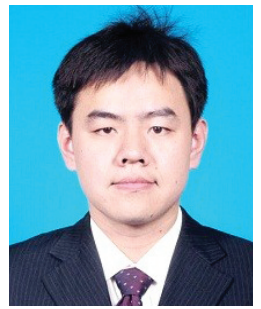

Congshi $\mathrm{Hu}$ received his B.S. and M.S. degrees in Electronic Engineering from Tsinghua University, Beijing, China, in 2011 and 2014 respectively. He is now a project manager in China Mobile Communications Corporation (CMCC). His research interests include green wireless communications and multiple antenna systems.

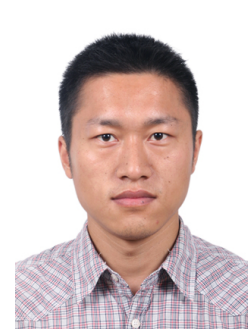

Jie Gong (S'09, M'13) received his B.S. and Ph.D. degrees in Department of Electronic Engineering in Tsinghua University, Beijing, China, in 2008 and 2013 , respectively. He is currently a postdoctorial scholar in Department of Electronic Engineering in Tsinghua University, Beijing, China. From July 2012 to January 2013, he visited Institute of Digital Communications, University of Edinburgh, Edinburgh, UK. He is a co-recipient of the Best Paper Award from IEEE Communications Society Asia-Pacific Board in 2013. His research interests include base station cooperation in cellular networks, energy harvesting and green wireless communications.

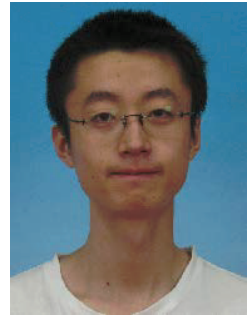

Xiaolei Wang (S'11) received his B.S. degree in Electronic Engineering from Tsinghua University, Beijing, China, in 2009. He is currently a Ph.D. student in Electronic Engineering Department at Tsinghua University, Beijing, China. His research interests cover green communications, content delivery network and energy harvesting 


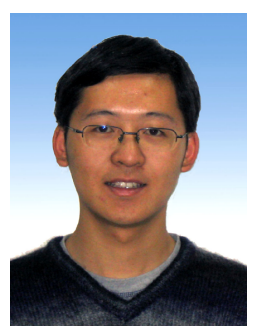

Sheng Zhou (S'06, M'12) received his B.E. and Ph.D. degrees in Electronic Engineering from Tsinghua University, Beijing, China, in 2005 and 2011 , respectively. He is now an assistant professor in Electronic Engineering Department at Tsinghua University, Beijing, China. From January to June 2010, he was a visiting student at Wireless System Lab, Electrical Engineering Department of Stanford University. $\mathrm{He}$ is a co-recipient of the Best Paper Award from the Asia-Pacific Conference on Communication (APCC) in 2009 and 2013, the 23th IEEE International Conference on Communication Technology (ICCT) in 2011, and the 25th Intl. Tele-traffic Cong. (ITC) in 2013. His research interests include cross-layer design for multiple antenna systems, cooperative transmission in cellular systems, and green wireless communications.

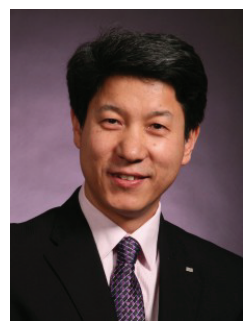

Zhisheng Niu (M'98-SM'99-F'12) graduated from Beijing Jiaotong University, China, in 1985, and got his M.E. and D.E. degrees from Toyohashi University of Technology, Japan, in 1989 and 1992, respectively. During 1992-94, he worked for Fujitsu Laboratories Ltd., Japan, and in 1994 joined with Tsinghua University, Beijing, China, where he is now a professor at the Department of Electronic Engineering and deputy dean of the School of Information Science and Technology. He is also a guest chair professor of Shandong University, China. His major research interests include queueing theory, traffic engineering, mobile Internet, radio resource management of wireless networks, and green communication and networks.

Dr. Niu has been an active volunteer for various academic societies, including Director for Conference Publications (2010-11) and Director for Asia-Pacific Board (2008-09) of IEEE Communication Society, Membership Development Coordinator (2009-10) of IEEE Region 10, Councilor of IEICEJapan (2009-11), and council member of Chinese Institute of Electronics (2006-11). He is now a distinguished lecturer (2012-15) and Chair of Emerging Technology Committee (2014-15) of IEEE Communication Society, a distinguished lecturer (2014-16) of IEEE Vehicular Technologies Society, a member of the Fellow Nomination Committee of IEICE Communication Society (2013-14), standing committee member of Chinese Institute of Communications (CIC, 2012-16), and associate editor-in-chief of IEEE/CIC joint publication China Communications.

Dr. Niu received the Outstanding Young Researcher Award from Natural Science Foundation of China in 2009 and the Best Paper Award from IEEE Communication Society Asia-Pacific Board in 2013. He also co-received the Best Paper Awards from the 13th, 15th and 19th Asia-Pacific Conference on Communication (APCC) in 2007, 2009, and 2013, respectively, International Conference on Wireless Communications and Signal Processing (WCSP'13), and the Best Student Paper Award from the 25th International Teletraffic Congress (ITC25). He is now the Chief Scientist of the National Basic Research Program (so called "973 Project") of China on "Fundamental Research on the Energy and Resource Optimized Hyper-Cellular Mobile Communication System" (2012-2016), which is the first national project on green communications in China. He is a fellow of both IEEE and IEICE. 